\title{
Bone marrow-derived mesenchymal stem cells promote healing of rabbit tibial fractures via JAK-STAT signaling pathway
}

\author{
PENG WANG and ZHIQIANG ZHANG
}

Department of Orthopaedics, Shandong Provincial Third Hospital, Jinan, Shandong 250031, P.R. China

Received October 18, 2019; Accepted December 17, 2019

DOI: $10.3892 /$ etm.2020.8441

\begin{abstract}
Influence of bone marrow-derived mesenchymal stem cells (BMMSCs) on the healing of rabbit tibial fractures and the role of the Janus kinase-signal transducer and activator of transcription (JAK-STAT) signaling pathway in fracture healing were explored. Rabbit BMMSCs were isolated and cultured in vitro, and their purity was determined using flow cytometry. Rabbit fracture models were established, and injected with BMMSCs, BMMSCs + TG101348 or TG101348, with those injected with an equal volume of normal saline as control group, and the repair of fracture ends was evaluated via X-ray examination 3 weeks later. The BMMSCs isolated in vitro grew well, and flow cytometry assay results showed that the positive expression rates of cluster of differentiation (CD)90 and CD105 in cells were 99.21 and $99.56 \%$, respectively, with no CD45 expressed. According to the results of CCK-8 assay, TG101348 lowered the proliferation level of BMMSCs, and the wound healing assay revealed that the migration ability of BMMSCs at 24 and $48 \mathrm{~h}$ was substantially weaker than that in control group $(\mathrm{P}<0.05)$. After induction of osteogenic differentiation for 3 weeks, alizarin red staining results manifested that osteogenic induction group had notably more calcium nodules than TG101348 group $(\mathrm{P}<0.05)$. Compared with those in control group, the protein expression levels of p-JAK2 and p-STAT3 were remarkably raised by osteogenic induction $(\mathrm{P}<0.05)$, but the protein expression levels of JAK2, p-JAK2 and p-STAT3 were considerably decreased by TG101348 $(\mathrm{P}<0.05)$. It was found through the $\mathrm{X}$-ray examination that the rabbits in control group and those injected with BMMSCs recovered well, and the latter had larger external calluses at fracture ends than the former, while the fracture ends of those injected with TG101348 and BMMSCs + TG101348 were not healed completely. BMMSCs promote healing of rabbit tibial fractures through the JAK-STAT signaling pathway.
\end{abstract}

Correspondence to: Dr Zhiqiang Zhang, Department of Orthopaedics, Shandong Provincial Third Hospital, 11 Wuyingshan Middle Road, Tianqiao, Jinan, Shandong 250031, P.R. China E-mail:pzgvu431@163.com

Key words: bone marrow-derived mesenchymal stem cells, the JAK-STAT signaling pathway, rabbit, fracture

\section{Introduction}

The tibia is the larger and stronger one of the two lower leg bones below the knee, and a tibial shaft fracture or breakage is a common orthopedic injury that may be attributed to road traffic accidents, falls from height and sports activities, with an annual morbidity rate of approximately $0.02 \%(1,2)$. Tibial fractures represent $1.66 \%$ of the total cases in adults, and nearly $10 \%$ of fracture patients have delayed healing or nonunion, which is the main complication in tibial fracture patients. In recent decades, as many as several million fracture patients are disabled due to poor healing $(3,4)$, so that the patients have decreased productivity and even lose working capacity, thereby increasing social cost $(5,6)$. Therefore, it is extremely important to study the physiological mechanism in the repair of fractures for facilitating fracture healing. The repair of fractures is an extremely complex physiological process that involves a wide variety of cells, including bone marrow-derived mesenchymal stem cells (BMMSCs), osteoprogenitors, osteoblasts and osteoclasts $(2,7)$, and osteoblasts, the critical functional cells derived from BMMSCs, are responsible for the metabolism and fracture healing of adult bones (7).

Mesenchymal stem cells (MSCs) are stem cells with the multi-directional differentiation potential, first discovered in bone marrows, and they can differentiate in vitro into such functional cells as osteoblasts, chondrocytes, lipocytes and myoblasts (8). Relevant studies have found that after fractures, BMMSCs can rapidly migrate into the fracture site and then start to proliferate and osteogenically differentiate. The osteogenic differentiation of BMMSCs is modulated by multiple hormones and local factors, so the stimulation of their osteogenic differentiation has been considered as an important mechanism by which fracture healing is accelerated $(9,10)$. The Janus kinase-signal transducer and activator of transcription (JAK-STAT) signaling pathway plays a vital role in multiple processes such as cell proliferation and differentiation. Several studies have demonstrated that the JAK-STAT pathway can regulate the function of osteoblasts and bone tissue regeneration and promote angiogenesis (11), while modulating the differentiation and migration of preosteoclasts (12).

The present study explored the role of the JAK-STAT signaling pathway in the osteogenic differentiation of BMMSCs and its influence on the repair of tibial fractures, providing theoretical bases for related research on fracture repair and offering potential clinical treatments. 


\section{Materials and methods}

Main materials. Rabbits aged 15 weeks, xylazine, ketamine and enrofloxacin, Dulbecco's modified Eagle's medium (DMEM), fetal bovine serum (FBS), phosphate buffered saline (PBS), and double antibodies (Gibco; Thermo Fisher Scientific, Inc.), cluster of differentiation (CD) 45-PE, CD90-PE, CD105-PE, JAK2, phosphorylated (p)-JAK2, p-STAT3 and $\beta$-actin antibodies (Abcam), TG101348 (Selleck Chemicals), and $0.22 \mu \mathrm{m}$ pinhole filter (EMD Millipore).

Establishment of rabbit fracture models. Rabbits underwent osteotomy and external fixation of the left middle tibia. Anesthesia was performed using xylazine $(2.5 \mathrm{mg} / \mathrm{kg})$ and ketamine $(22 \mathrm{mg} / \mathrm{kg})$ and maintained with isoflurane. Then the animals were placed in right lateral position, and the left pelvis and limb were prepared for operation. A $1 \mathrm{~cm}$-long cranial lateral incision was made at the tibial shaft, and the skull tibial muscle and gastrocnemius muscle were bluntly anatomized and separated to expose the tibia which was then fixed using a mini fixator. With the lateral tibial periosteum longitudinally cut open, transverse osteotomy was conducted using a saw, and sterile saline was used for continuous flushing. Subsequently, four needles with a diameter of $1.6 \mathrm{~mm}$ were led into the lateral middle tibial shaft, and the fracture site was fixed using two proximal needles and the distal needles in tibial osteotomy. Finally, the fixator was fixed into the pin for routine muscular and subcutaneous closure. A preventive dose of enrofloxacin $(5 \mathrm{mg} / \mathrm{kg})$ was subcutaneously administered before operation and at 3 days after operation. This study was approved by the Animal Ethics Committee of Shandong Provincial Third Hospital Animal Center (Jinan, China).

Isolation and culture of BMMSCs. The tibia of rabbits was removed under sterile conditions, and cleaned using PBS. Then the bone ends were sawed off, and the bone cavity was rinsed with the DMEM containing 10\% FBS and 1\% double antibodies using a syringe. The cell suspension was harvested, centrifuged at $4^{\circ} \mathrm{C}, 1,050 \mathrm{x}$ for $5 \mathrm{~min}$, added with an appropriate amount of DMEM containing $10 \%$ FBS and $1 \%$ double antibodies for re-suspension and sedimentation. Following counting, the cells were inoculated into a $100 \mathrm{~mm}$ culture dish at the density of $1 \times 10^{5}$ cells $/ \mathrm{ml}$ and cultured in an incubator containing $5 \% \mathrm{CO}_{2}$ at $37^{\circ} \mathrm{C}$. The medium was replaced every other day until $90 \%$ of the dish bottom was covered. The resulting cells were washed using PBS twice, digested by $0.25 \%$ trypsin and sub-cultured at the density of 1:3.

Cell injection. BMMSCs were amplified through in vitro culture, and passage 5 (P5) BMMSCs growing well were collected and prepared into the cell suspension at the density of $1 \times 10^{7}$ cells $/ \mathrm{ml}$. The fracture rabbits prepared as above were divided into control group, inhibitor group, BMMSC injection group and BMMSCs + inhibitor group. The fracture end was locally injected with $500 \mu \mathrm{l}$ of the cell suspension in BMMSC injection group, $500 \mu \mathrm{l}$ of the cell suspension was locally injected into the fracture end, and $100 \mathrm{mg} / \mathrm{kg}$ TG101348 was orally taken in BMMSCs + inhibitor group, an equal volume of normal saline was locally injected into the fracture end, and
$100 \mathrm{mg} / \mathrm{kg}$ TG101348 was orally taken in inhibitor group, and the fracture end was locally injected with $500 \mu 1$ of normal saline in control group.

Cell Counting Kit (CCK)-8 assay. The P3 BMMSCs with good growth were seeded into a $96-$ well plate at $1 \times 10^{4}$ cells $/ \mathrm{ml}$ and cultured using DMEM + 10\% FBS or DMEM + 10\% FBS $+1 \mu \mathrm{M}$ of TG101348 for 7 days. The corresponding wells were added with $10 \mu \mathrm{l}$ of CCK-8 solution daily (Dojindo Molecular Technologies, Inc.), and the culture plate was incubated in an incubator for 1-2 h. Finally, the absorbance at $450 \mathrm{~nm}$ was determined using a microplate reader.

Wound healing assay. The P3 BMMSCs growing well were inoculated into a 6 -well plate at $2 \times 10^{5}$ cells $/ \mathrm{ml}$ and cultured using DMEM + 10\% FBS until the cells completely covered the dish bottom, and a straight scratch was made using a pipette tip. The cells were washed using PBS 3 times. With the scratched cells removed, the resulting cells were cultured in DMEM or DMEM $+1 \mu \mathrm{M}$ of TG101348, and they were photographed and analyzed at 24 and $48 \mathrm{~h}$.

Osteogenic induction of BMMSCs. The P3 BMMSCs growing well were seeded into a 6 -well plate at $2 \times 10^{5}$ cells $/ \mathrm{ml}$ and started to be osteogenically induced when $90 \%$ of the dish bottom was covered by the cells using the following medium: $\mathrm{DMEM}+50 \mathrm{mg} / \mathrm{l}$ ascorbic acid $+0.5 \mathrm{mM}$ of sodium $\beta$-glycerophosphate $+1 \mu \mathrm{M}$ of dexamethasone and $10 \% \mathrm{FBS}$. The BMMSCs cultured in osteogenic induction medium were taken as induction group, those in osteogenic induction medium $+1 \mu \mathrm{M}$ of TG101348 as inhibitor group, and those in $\mathrm{DMEM}+10 \% \mathrm{FBS}$ as control group, and they were cultured in an incubator containing $5 \% \mathrm{CO}_{2}$ at $37^{\circ} \mathrm{C}$. The medium was changed every other day, and the culture lasted for 3 weeks.

Flow cytometry analysis. Flow cytometry analysis was performed in the P3 BMMSCs growing well as follows. After being washed using PBS twice, the cells were digested using $0.25 \%$ trypsin into single-cell suspension, and rinsed using PBS 3 times. With the density adjusted to $3 \times 10^{5}$ cells $/ \mathrm{ml}$, the cells were incubated separately with CD45-PE, CD90-PE and CD105-PE antibodies for flow cytometry in the dark at $4^{\circ} \mathrm{C}$, and the cell samples incubated with anti-rat IgG1-PE antibody were taken as homotype controls. The resulting cells were washed using PBS 3 times, and detected with a flow cytometer (Beckman Coulter, Inc.). Finally, the data were analyzed using Kaluza software.

Alizarin red staining and semi-quantitative analysis. After the induction of osteogenic differentiation for 3 weeks, the cells were rinsed with PBS twice, fixed in an appropriate volume of $4 \%$ paraformaldehyde at room temperature, washed with PBS twice again and incubated with $40 \mathrm{mM}$ of alizarin red staining solution at $37^{\circ} \mathrm{C}$ for $30 \mathrm{~min}$. With the staining solution aspirated, PBS was added to wash the cells 3 times, and the cells were observed and photographed under a microscope. After PBS was discarded, the calcium nodules were dissolved using $10 \mathrm{mM}$ of cetylpyridinium chloride. Finally, the absorbance at a wavelength of $550 \mathrm{~nm}$ was measured using the microplate reader for semi-quantitative analysis. 

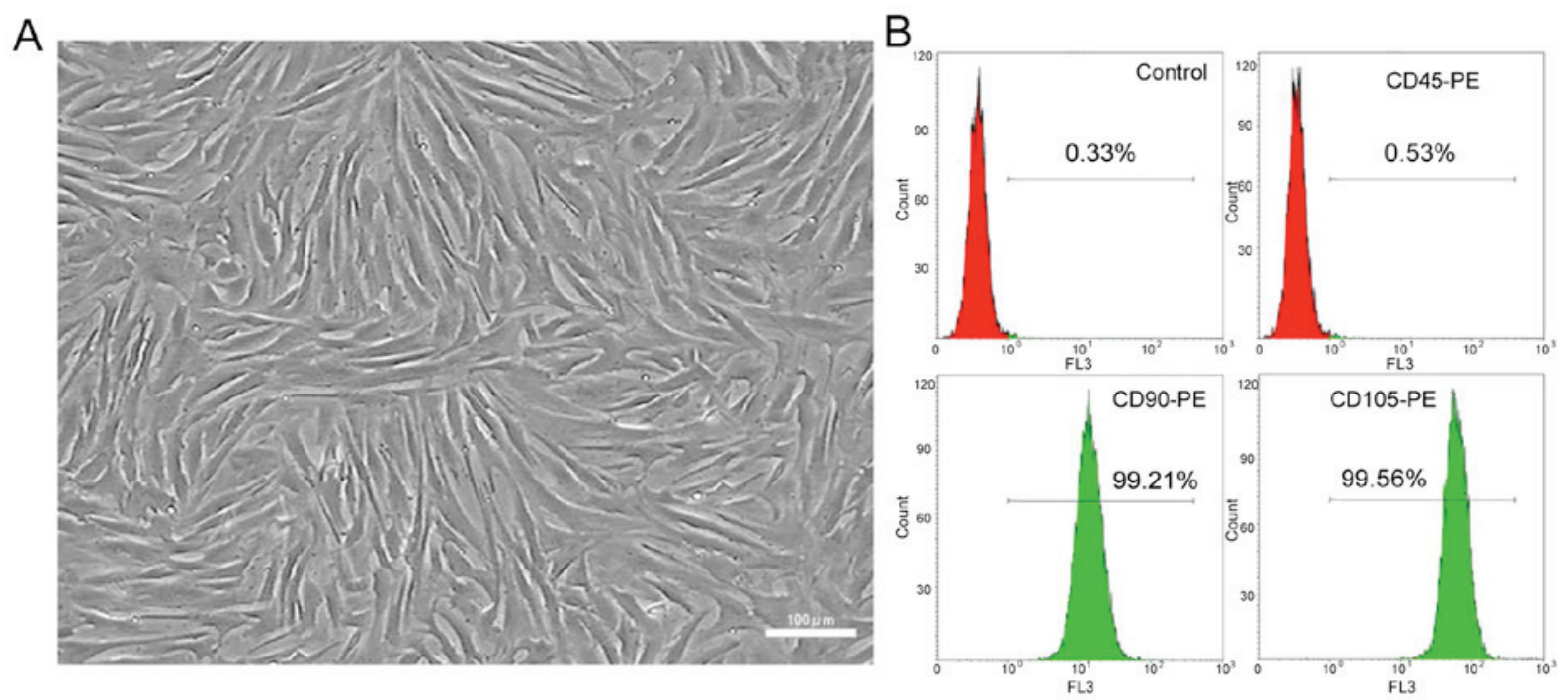

Figure 1. Culture and flow cytometry identification and analysis of rabbit BMMSCs. (A) Morphology of BMMSCs under an inverted microscope. (B) Results of flow cytometry analysis: The positive expression rates of CD45, CD90 and CD105 in cells are 0.53, 99.21 and $99.56 \%$, respectively. Scale bar $100 \mu \mathrm{m}$. BMMSCs, bone marrow-derived mesenchymal stem cells; $\mathrm{CD}$, cluster of differentiation.

Western blotting. The cells induced to differentiate and cultured in vitro were added with cell lysis buffer in moderation. After the cell lysis suspension was collected using a cell scraper, the cells were lysed at $4^{\circ} \mathrm{C}$ overnight, and centrifuged at $4^{\circ} \mathrm{C}$, $11,500 \mathrm{x}$ g for $10 \mathrm{~min}$ to extract total proteins. Then the concentration of the proteins was determined via bicinchoninic acid (BCA) protein assay (Pierce; Thermo Fisher Scientific, Inc.), and the proteins were separated through $8 \%$ sodium dodecyl sulphate-polyacrylamide gel electrophoresis (SDS-PAGE) and transferred onto a polyvinylidene fluoride (PVDF) membranes (EMD Millipore). Subsequently, the membrane was sealed in Tris buffered saline using 5\% skim milk powder and $0.1 \%$ Tween-20 and slightly shaken and reacted with the JAK2, p-JAK2, p-STAT3 and $\beta$-actin primary antibodies at $4^{\circ} \mathrm{C}$ overnight. Afterwards, the horseradish peroxidase (HRP)-labeled secondary antibodies were added for incubation. Finally, the proteins were treated with electrochemiluminescence (ECL) reagent, exposed and detected.

$X$-ray examination. The fracture site was examined using an $\mathrm{X}$-ray machine through the perspective shooting, with the tube projection distance of $100 \mathrm{~cm}$, and at $100 \mathrm{~mA}$ and $50 \mathrm{kV}$, immediately after the establishment of rabbit fracture models and at 3 weeks after operation, so as to observe the fracture models and postoperative and post-treatment fractures. Finally, X-ray images were analyzed using medical image analysis software.

Statistical analysis. Statistical Product and Service Solutions (SPSS) 20.0 software (IBM Corp.) was applied to statistically process data. The data in each group were expressed as mean \pm standard deviation (mean $\pm \mathrm{SD}$ ), and inter-group comparison was made using independent-samples t-test. $\mathrm{P}<0.05$ was considered to indicate a statistically significant difference.

\section{Results}

In vitro isolation and culture of rabbit BMMSCs. The BMMSCs isolated and cultured in vitro showed favorable and

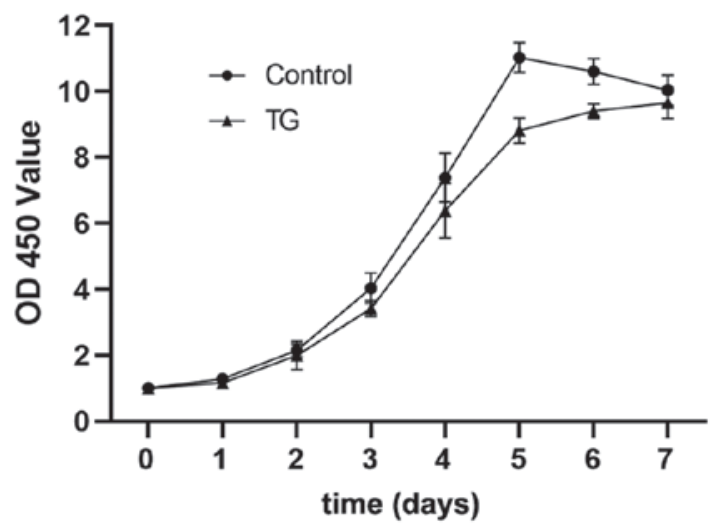

Figure 2. OD of BMMSCs at 0, 1, 2, 3, 4, 5, 6 and 7 days determined via CCK-8 assay. Control, BMMSCs normally cultured; TG, those cultured in the medium added with JAK2 inhibitor TG101348. BMMSCs, bone marrowderived mesenchymal stem cells.

whirlpool-like growth, and plumpness in morphology (Fig. 1A). According to the results of flow cytometry analysis in $\mathrm{P} 3$ cells, the positive expression rates of $\mathrm{CD} 45, \mathrm{CD} 90$ and $\mathrm{CD} 105$ in cells were $0.53,99.21$ and $99.56 \%$, respectively (Fig. 1B).

Proliferation and wound healing results of BMMSCs. BMMSCs were cultured using JAK2 inhibitor TG101348, with those normally cultured as controls. At $0,1,2,3,4,5,6$ and 7 days, the optical density (OD) was measured using CCK- 8 assay, and it was found that the cells proliferated in a sigmoid curve, while the application of TG101348 lowered the proliferation level of BMMSCs (Fig. 2). The wound healing assay results revealed that compared with those in control group, the BMMSCs cultured with TG101348 had a notably weakened migration ability at 24 and $48 \mathrm{~h}(\mathrm{P}<0.05)$ (Fig. 3).

In vitro induction of osteogenic differentiation of BMMSCs. BMMSCs were induced in vitro to differentiate into osteoblasts for 3 weeks, and the alizarin red staining results showed 
A
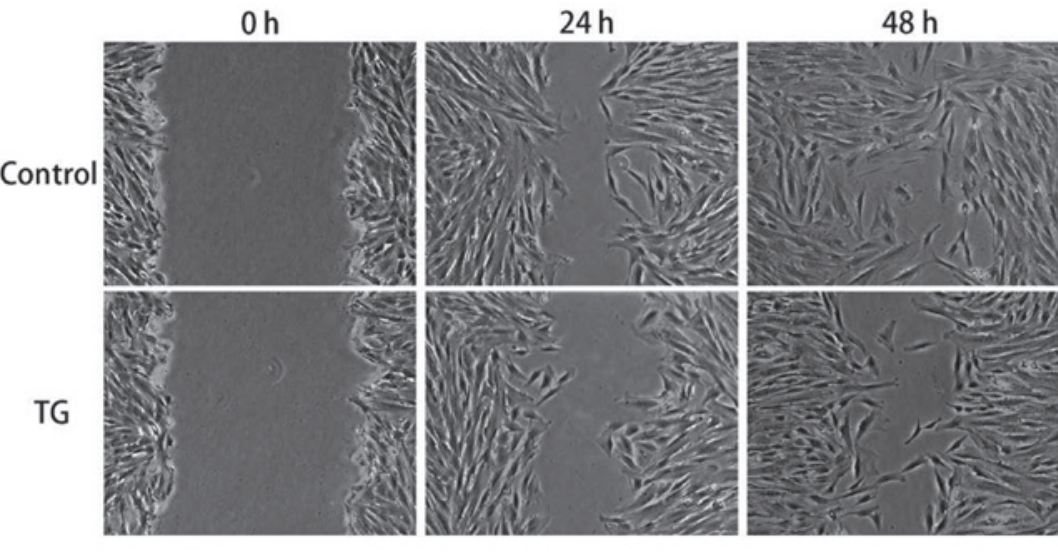

B

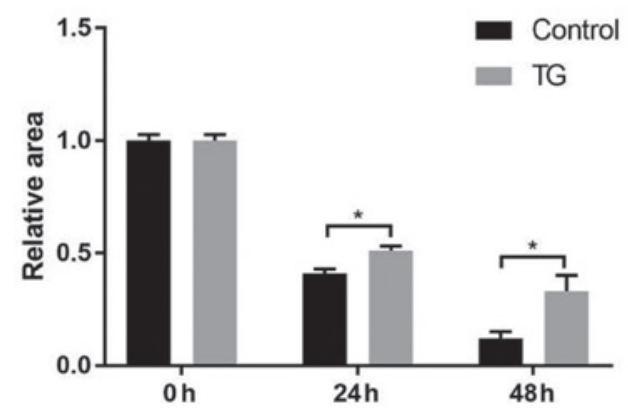

Figure 3. Wound healing assay results. (A) Migration of differentially treated cells at 0,24 and $48 \mathrm{~h}$. (B) Relative quantification of the area of the culture dish uncovered by cells: The migration ability of BMMSCs cultured with TG101348 at 24 and $48 \mathrm{~h}$ is remarkably weaker than that in control group $(\mathrm{P}<0.05) .{ }^{*} \mathrm{P}<0.05$, the difference is statistically significant. Control, BMMSCs normally cultured; TG, those cultured in the medium added with JAK2 inhibitor TG101348. BMMSCs, bone marrow-derived mesenchymal stem cells.

A

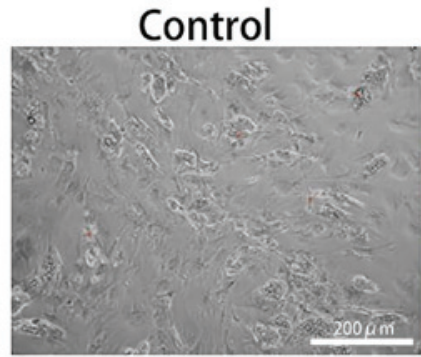

B
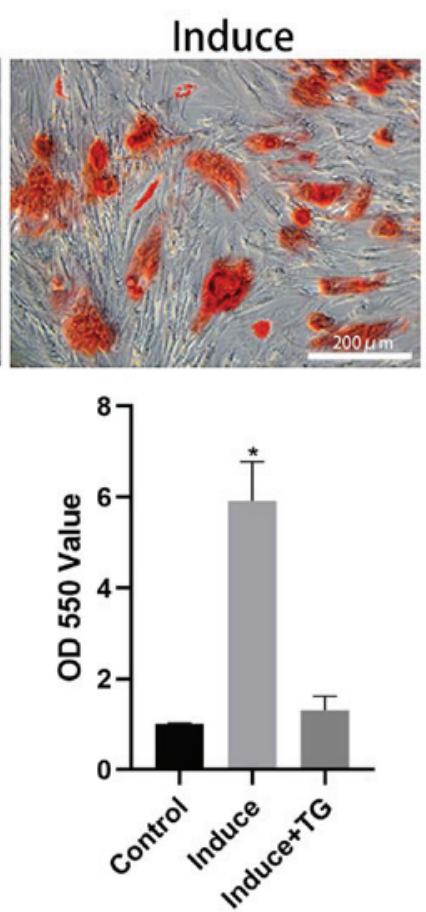

Induce+TG

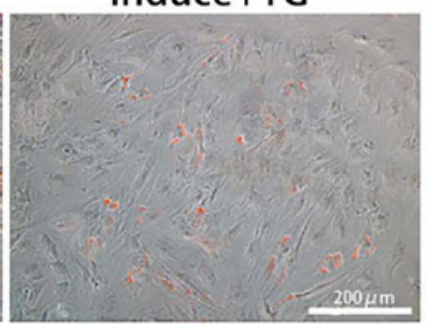

Figure 4. In vitro induction of differentiation of BMMSCs into osteoblasts. (A) Alizarin red staining results of BMMSCs after induction of osteogenic differentiation. (B) Semi-quantitative analysis of the alizarin red staining results using a microplate reader at $550 \mathrm{~nm}$ : The osteogenically induced BMMSCs exhibit a considerably higher OD value than those co-induced with TG101348 ( $\mathrm{P}<0.05)$. "P<0.05, difference is statistically significant compared with the other two groups. Control, BMMSCs normally cultured; Induce, BMMSCs osteogenically induced; Induce + TG, BMMSCs osteogenically induced with JAK inhibitor TG101348. BMMSCs, bone marrow-derived mesenchymal stem cells; OD, optical density.

that the osteogenically induced BMMSCs had large numbers of calcium nodules, while only few of those co-induced with
JAK2 inhibitor TG101348 were stained red, but the BMMSCs normally cultured for 3 weeks were not stained red (Fig. 4A). 
A

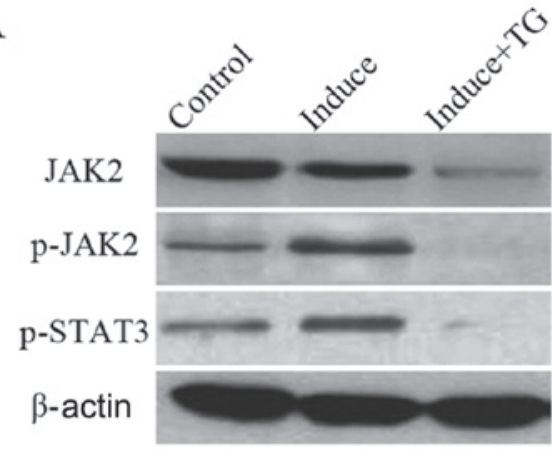

B

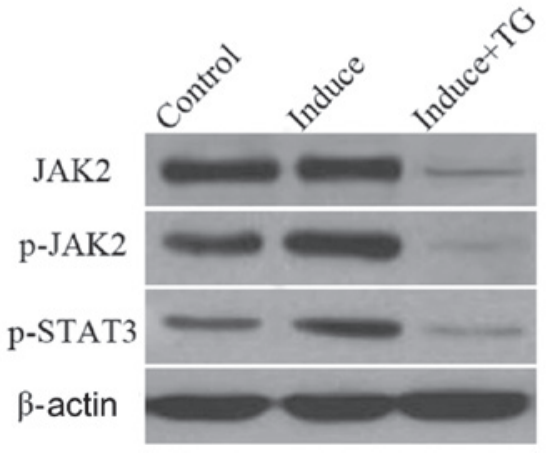

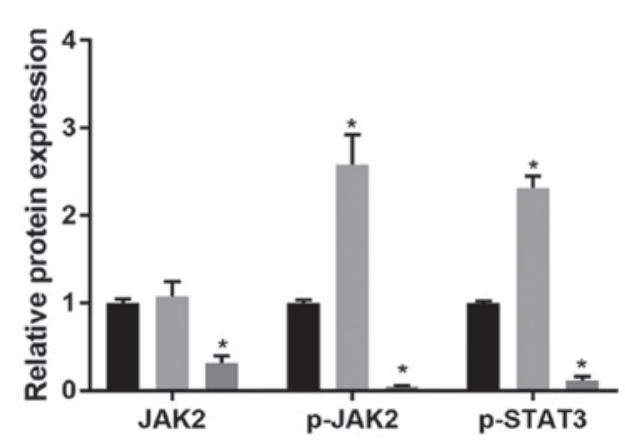

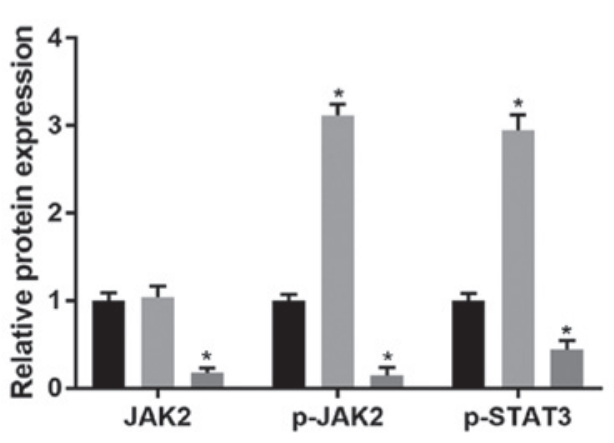

- Control

Induce

Induce+TG

Figure 5. The JAK-STAT signaling pathway in the induction of osteogenic differentiation of BMMSCs detected via WB. (A) Protein expression of JAK2, p-JAK2 and p-STAT3 in the 1st week of the induction: Compared with those in control group, the protein expression levels of p-JAK2 and p-STAT3 are substantially elevated in induction group $(\mathrm{P}<0.05)$, while the expressions of JAK2, p-JAK2 and p-STAT3 are suppressed by TG101348 ( $<<0.05)$. (B) Protein expression of JAK2, p-JAK2 and p-STAT3 in the 3rd week of the induction: The protein expression levels of p-JAK2 and p-STAT3 in osteogenic induction group are notably higher than those in control group $(\mathrm{P}<0.05)$, whereas the protein expression levels of JAK2, p-JAK2 and p-STAT3 are lowered by TG101348 compared with those in control group $(\mathrm{P}<0.05)$. ${ }^{*} \mathrm{P}<0.05$, the differences are statistically significant compared with the other two groups. Control, BMMSCs normally cultured; Induce, BMMSCs normally cultured; Induce + TG, BMMSCs osteogenically induced with JAK inhibitor TG101348. JAK-STAT, Janus kinase-signal transducer and activator of transcription; BMMSCs, bone marrow-derived mesenchymal stem cells; WB, western blotting; p-, phosphorylated.

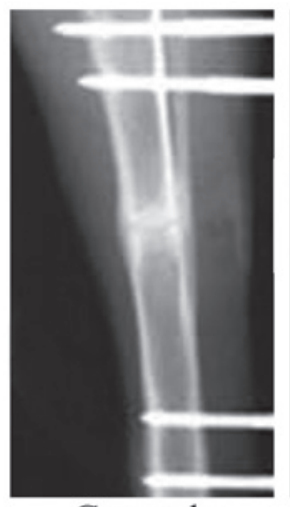

Control

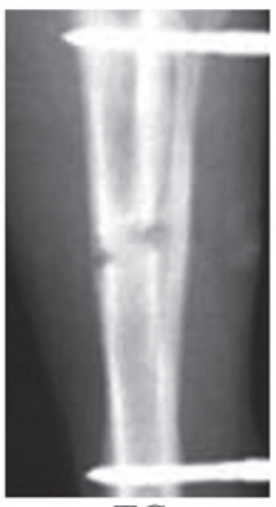

TG

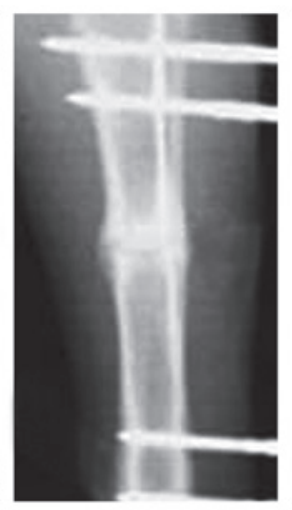

BMMSCs

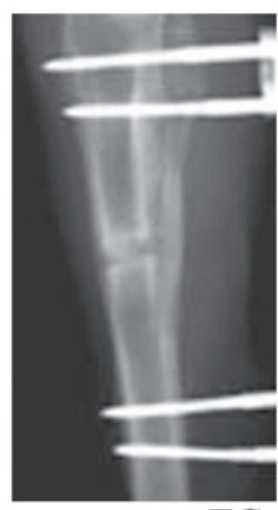

BMMSCs+TG

Figure 6. X-ray examination results of fracture ends in rabbits differently treated at 3 weeks after fractures. BMMSCs, bone marrow-derived mesenchymal stem cells; TG, TG101348.

It was found through the semi-quantitative analysis of the alizarin red staining results that the OD of osteogenically induced BMMSCs was notably higher than that of the BMMSCs co-induced with TG101348 ( $\mathrm{P}<0.05)$ (Fig. 4B).

Analysis of JAK/STAT signaling pathway in osteogenic induction. BMMSCs were induced to osteogenically differentiate for 1 or 3 weeks, and then the JAK-STAT signaling pathway-related proteins JAK2, p-JAK2 and p-STAT3 were detected using WB. The results showed that compared with those in control group, the protein expression levels of p-JAK2 and p-STAT3 increased notably in induction group at 1 and 3 weeks $(\mathrm{P}<0.05)$, whereas the protein expressions of JAK2, p-JAK2 and p-STAT3 in the osteogenic induction of cells were considerably repressed by JAK2 inhibitor TG101348 $(\mathrm{P}<0.05)$. As the time of induction was extended, the expression levels of p-JAK2 and p-STAT3 in the 3rd week of induction were higher than those in the 1st week (Fig. 5).

Role of BMMSC transplantation in the healing of rabbit fractures. After tibial fractures, the rabbits were injected with BMMSCs, JAK2 inhibitor TG101348 or BMMSCs + TG101348, 
and 3 weeks later, the recovery of fractures was evaluated via X-ray examination. Based on the results, the rabbits in control group and those injected with BMMSCs recovered well. Moreover, the external calluses at the fracture ends of the rabbits injected with BMMSCs were larger than those in control group, while the fracture ends were not completely healed in the rabbits injected with JAK2 inhibitor TG101348 or BMMSCs + TG101348 (Fig. 6).

\section{Discussion}

Fracture healing is a complex physiological process in which the bone is locally repaired. This process involves such cells as osteoblasts and osteoclasts that are directly implicated in bone remodeling and bone resorption, and massive osteoblasts produced in the early stage of healing is vital for the repair of fractures (13). Relevant studies have corroborated that after fractures, MSCs migrate to the fracture site to proliferate and differentiate into osteoblasts. The mature osteoblasts secrete a matrix material called osteoid, and then they are embedded in the osteoid and differentiate into osteocytes, followed by the formation of bone matrices via calcification. Ultimately, the healing of fractures end up with the formation of bone tissues $(13,14)$. BMMSCs play a crucial role in the whole repair process of fractures (9). Stem cells have been applied to the treatment of fractures, such as nonunion (15), and the transplantation of culture-amplified BMMSCs can enhance local repair (7). In this study, the fracture site was locally injected with BMMSCs, and it was discovered that they promoted the formation of external calluses and repair of fractures.

The osteoblasts and osteoclasts in bone development and repair can be modulated by various local factors, including cytokines in bone microenvironment and multiple cytokine signaling pathways, such as the JAK-STAT pathway. The JAK-STAT signaling pathway was originally identified as a pathway that mainly responds to the activation of receptors of interferon- $\gamma$ and interleukin- 6 family members (16). The JAK family members comprise JAK1, JAK2, JAK3 and Tyk2, and STAT protein was initially discovered as the potential cytoplasmic transcription factor (17). The phosphorylation of STATs is triggered by the binding of cytokines to their receptors on the cell surface via the JAK-STAT signaling pathway (18). The JAK-STAT signaling pathway plays a key role in the growth and differentiation of various types of cells, and many cytokines that activate this pathway have been found through studies to affect the proliferation and differentiation of osteoblasts and osteoclasts. The in vitro activation of the JAK-STATsignaling pathway can enhancealkaline phosphatase activity and increase osteocalcin expression, suggesting that the differentiation of osteoblasts can be promoted (19). In this study, the expression of JAK in BMMSCs was inhibited by the JAK inhibitor, and it was discovered that the proliferation and migration abilities of cells were weakened, implying that the JAK-STAT signaling pathway has a certain effect on BMMSCs. A previous study has demonstrated that STAT3 can promote proliferation and enhance anti-inflammatory activity, and that the JAK inhibitor inhibits the expression of JAK2 and weakens the activity of STAT3, thereby leading to the decline in the proliferative ability of cells (19). The osteogenic differentiation of BMMSCs was further induced, and the results revealed that JAK inhibitor substantially inhibited the generation of osteoblasts, illustrating that the JAK2-STAT3 signaling pathway is important in the differentiation of stem cells into osteoblasts.

According to a study (20), JAK2 deficiency causes the decoupling between grow th hormone-receptor signals and the downstream mediator STAT, thus affecting the normal development of bones. Since STAT3 mediates the anabolism signals in osteoblasts and regulates bone formation, selectively inactivating STAT3 in osteoblasts can inhibit bone formation, thereby decreasing the bone mass and raising the incidence rate of traumatic fractures in individuals $(21,22)$. Additionally, prior to bone formation, the mice with JAK2 knocked out die of anemia at E12.5 (20). The fracture sites were injected with BMMSCs in this study, and it was found that the degree of the repair of fractures was increased, whereas the healing capacity of fractures declined in the presence of JAK inhibitor, probably because the JAK inhibitor suppresses the expression of JAK2, causing the decoupling of its downstream mediator STAT3, inhibiting the differentiation of MSCs into osteoblasts and the expression of anabolism signals in osteoblasts (17), and ultimately weakening the healing capacity of fractures.

Based on the results of this study, BMMSCs can differentiate into osteoblasts in vitro and promote the repair of fractures and the mineralization of bone tissues in the fracture healing probably through the JAK-STAT signaling pathway.

\section{Acknowledgements}

Not applicable.

\section{Funding}

No funding was received.

\section{Availability of data and materials}

All data generated or analyzed during this study are included in this published article.

\section{Authors' contributions}

PW and ZZ designed the study and performed the experiments, collected the data, analyzed the data and prepared the manuscript. All authors read and approved the final manuscript.

\section{Ethics approval and consent to participate}

This study was approved by the Animal Ethics Committee of Shandong Provincial Third Hospital Animal Center (Jinan, China).

\section{Patient consent for publication}

Not applicable.

\section{Competing interests}

The authors declare that they have no competing interests. 


\section{References}

1. Court-Brown CM and Caesar B: Epidemiology of adult fractures: A review. Injury 37: 691-697, 2006.

2. Lillo M, El Ezzo O, Cauteruccio M, Ziranu A, De Santis V and Maccauro G: Infections in primary intramedullary nailing of open tibial fractures: A review article. Eur Rev Med Pharmacol Sci 23 (Suppl): 195-200, 2019.

3. GamalO and Shams A: Surgical technique for biological fixation of closed segmental tibial fractures by the Less Invasive Stabilization System (LISS). SICOT J 4: 48, 2018

4. Kadar A, Sherman H, Glazer Y, Katz E and Steinberg EL: Predictors for nonunion, reoperation and infection after surgical fixation of patellar fracture. J Orthop Sci 20: 168-173, 2015.

5. Antonova E, Le TK, Burge R and Mershon J: Tibia shaft fractures: Costly burden of nonunions. BMC Musculoskelet Disord 14: 42, 2013.

6. Goh EL, Chidambaram S, Eigenmann D, Ma S and Jones GG: Minimally invasive percutaneous plate osteosynthesis versus intramedullary nail fixation for closed distal tibial fractures: A meta-analysis of the clinical outcomes. SICOT J 4: 58, 2018.

7. Bajada S, Harrison PE, Ashton BA, Cassar-Pullicino VN, Ashammakhi N and Richardson JB: Successful treatment of refractory tibial nonunion using calcium sulphate and bone marrow stromal cell implantation. J Bone Joint Surg Br 89: 1382-1386, 2007.

8. Ji M, Bai C, Li L, Fan Y, Ma C, Li X and Guan W: Biological characterization of sheep kidney-derived mesenchymal stem cells. Exp Ther Med 12: 3963-3971, 2016.

9. Taguchi K, Ogawa R, Migita M, Hanawa H, Ito $\mathrm{H}$ and Orimo H: The role of bone marrow-derived cells in bone fracture repair in a green fluorescent protein chimeric mouse model. Biochem Biophys Res Commun 331: 31-36, 2005.

10. Khosla S, Westendorf JJ and Oursler MJ: Building bone to reverse osteoporosis and repair fractures. J Clin Invest 118: 421-428, 2008

11. Kamran MZ, Patil P and Gude RP: Role of STAT3 in cancer metastasis and translational advances. BioMed Res Int 2013: 421821,2013
12. Li DQ, Wan QL, Pathak JL and Li ZB: Platelet-derived growth factor BB enhances osteoclast formation and osteoclast precursor cell chemotaxis. J Bone Miner Metab 35: 355-365, 2017.

13. Gu Y, Zhou J, Wang Q, Fan W and Yin G: Ginsenoside Rg1 promotes osteogenic differentiation of rBMSCs and healing of rat tibial fractures through regulation of GR-dependent BMP-2/ SMAD signaling. Sci Rep 6: 25282, 2016.

14. Geris L, Gerisch A, Sloten JV, Weiner R and Oosterwyck HV Angiogenesis in bone fracture healing: A bioregulatory model. J Theor Biol 251: 137-158, 2008.

15. Lee EH and Hui JH: The potential of stem cells in orthopaedic surgery. J Bone Joint Surg Br 88: 841-851, 2006.

16. Li J: JAK-STAT and bone metabolism. JAK-STAT 2: e23930, 2013.

17. Darnell JE Jr: STATs and gene regulation. Science 277: 1630-1635, 1997.

18. Heinrich PC, Behrmann I, Müller-Newen G, Schaper F and Graeve L: Interleukin-6-type cytokine signalling through the gp130/Jak/STAT pathway. Biochem J 334: 297-314, 1998.

19. Bellido T, Borba VZ, Roberson P and Manolagas SC: Activation of the Janus kinase/STAT (signal transducer and activator of transcription) signal transduction pathway by interleukin-6-type cytokines promotes osteoblast differentiation. Endocrinology 138: 3666-3676, 1997.

20. Parganas E, Wang D, Stravopodis D, Topham DJ, Marine JC, Teglund S, Vanin EF, Bodner S, Colamonici OR, van Deursen JM, et al: Jak2 is essential for signaling through a variety of cytokine receptors. Cell 93: 385-395, 1998.

21. Bromberg J: Stat proteins and oncogenesis. J Clin Invest 109: 1139-1142, 2002.

22. Grimbacher B, Holland SM, Gallin JI, Greenberg F, Hill SC, Malech HL, Miller JA, O'Connell AC and Puck JM: Hyper-IgE syndrome with recurrent infections - an autosomal dominant multisystem disorder. N Engl J Med 340: 692-702, 1999.

This work is licensed under a Creative Commons Attribution-NonCommercial-NoDerivatives 4.0 International (CC BY-NC-ND 4.0) License. 